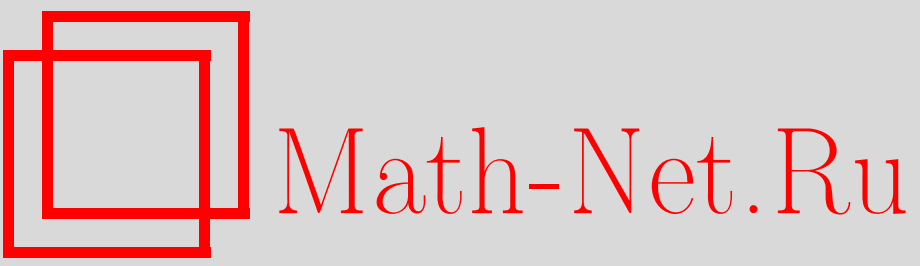

С. П. Струнков, К.-П. Шум, Об одной проблеме бернсайдовского типа для конечных полугрупп, УМН, 1998, том 53, выпуск 1, 227-228

DOI: https://doi.org/10.4213/rm29

Использование Общероссийского математического портала Math-Net.Ru подразумевает, что вы прочитали и согласны с пользовательским соглашением

http://www.mathnet.ru/rus/agreement

Параметры загрузки:

IP : 3.85 .5 .30

26 апреля 2023 г., 11:09:32 


\title{
ОБ ОДНОЙ ПРОБЛЕМЕ БЕРНСАЙДОВСКОГО ТИПА ДЛЯ КОНЕЧНЫХ ПОЛУГРУПП
}

\author{
С. П. Струнков, К. П. Шум
}

Вопросы о возможности восстановить или почти восстановить алгебраический объект по каким-либо его свойствам или системам параметров занимают важное место в алгебре, решения подобных вопросов обогащают алгебру новыми идеями и методами. В ряду этих задач заметно выделяется ослабленная проблема Бернсайда (ОПБ) о возможности почти восстановить конечную группу по числу образующих и показателю [1]. Решение этой проблемы Е. И. Зельмановым [3], [4] для $p$-групп представляет собой заметный вклад в теорию конечных групп. В настоящей работе рассматривается одна задача о возможности почти восстановить конечную полугруппу по некоторой системе ее параметров. Одним из этих параметров является число образующих полугруппы, поэтому эта задача похожа на ОПБ для групп. Положительное решение этой задачи дает возможность сформулировать аналог ОПБ для полугрупп.

Пусть $S=\left\{a_{1}, \ldots, a_{s}\right\}$-конечная полугруппа, $V$-линейное пространство размерности $s+1$ над полем комплексных чисел $\mathbb{C}$ и $e_{\varepsilon}, e_{a_{1}}, \ldots, e_{a_{s}}$ - некоторый базис пространства $V$. Для любого элемента $a \in S$ определим линейный оператор $L_{a}$ пространства $V$ при помощи следующих соотношений:

$$
L_{a}\left(e_{\varepsilon}\right)=e_{a}, \quad L_{a}\left(e_{a_{i}}\right)=e_{a a_{i}}, \quad i=1, \ldots, s .
$$

Действие оператора $L_{a}$ на остальных векторах пространства $V$ определяется естественным образом по линейности. В [2] показано, что соответствие $a \rightarrow L_{a}$ является изоморфизмом полугруппы $S$ на полугруппу линейных операторов $\left\{L_{a} \mid a \in S\right\}$. Это соответствие дает возможность рассматривать линейные комбинации элементов полугруппы (т.е. элементы полугрупповой алгебры $A(\mathbb{C} S)$ ), а также их собственные значения и спектр. Под индексом нильпотентности произвольного линейного оператора $L$ конечномерного комплексного пространства (и, тем самым, элемента полугрупповой алгебры) мы понимаем индекс нилпотентности его нильпотентной части $N$ в жордановом разложении $L=D+N$ оператора $L$ на сумму диагональной части $D$ и нильпотентной $N$.

Для элементов $a$ и $b$ конечной полугруппы $S$ обозначим через $p(a, b)$ число решений уравнения $a x=b$ в $S$.

Следующая теорема выявляет некоторые параметры, позволяющие почти определить конечную полугруппу. Аналогичный результат для групп был доказан ранее в [5].

Теорема. Пусть $n, \alpha_{1}, \ldots, \alpha_{n}, d, l, p u q$ - произвольные натуральные числа и $\Sigma$ множество попарно неизоморфных конечных полугрупп, порожденных $n$ образующими. Предположим, что для каждой полугруппь $S=\left\langle g_{1}, \ldots, g_{n}\right\rangle$ множества $\Sigma$ выполняются следующие условия:

1) алгебраическая степень каждого числа спектра әлемента $g=\alpha_{1} g_{1}+\cdots+\alpha_{n} g_{n}$ алгебры $A(\mathbb{C} S)$ не превосходит числа $d ;$

2) индекс нильпотентности элемента $g$ не больше числа $l$;

3) $p\left(g_{i}, b\right) \leqslant p, i=1, \ldots, n$, для любого әлемента $b \in S$;

4) число минимальных левых идеалов полугруппы $S$ не превосходит числа $q$.

Тогда мно жество г конечно.

В доказательстве теоремы существенную роль играют следующие утверждения, представляющие самостоятельный интерес.

This research is partially supported by a small project grant \#216026 of CUHK, Hong Kong, the U.S. Civilian Research and Development Foundation under Award No. RM1-346 and RFBR (\#96-01-00931). 
Лемма 1. Число попарно различных собственных значений әлемента $g \in S$ может быть ограничено сверху числом, зависящим от $n, \alpha_{1}, \ldots, \alpha_{n}, d$ (и не зависящим от выбора полугруппь $S$ ).

ЛЕмма 2. Порядок минимального левого идеала полугруппь $S$ может быть ограничен сверху числом, зависящим от $n, \alpha_{i}, \ldots, \alpha_{n}, d, l, p$ (и не зависящим от выбора полугруппь $S$ ).

Теорема настоящей работы позволяет сфформулировать следующий аналог ослабленной проблемы Бернсайда для конечных полугрупп. Пусть $n, d, l, p$ и $q$ - произволшные натуралшные числа и $\Sigma$ - множество попарно неизоморфных конечных полугрупп, порожденных $n$ образующими. Предположим, что для каждой полугруппы $S$ множества $\Sigma$ выполняются следующие условия:

1) алгебраические степени чисел спектра каждого элемента полугруппы $S$ не превосходят числа $d$;

2) индекс нильпотентности каждого элемента полугруппы $S$ не больше числа $l$;

3) $p(a, b) \leqslant p$ для любых элементов $a, b \in S$;

4) число минимальных левых идеалов полугруппы $S$ не превосходит числа $q$.

Является ли множество $\Sigma$ конечньц?

\section{СПИСОК ЛИТЕРАТУРЫ}

[1] Кострикин А. И. Вокруг Бернсайда. М.: Наука, 1986. [2] Ляпин Е. С. Полугруппы. М.: Физматгиз, 1959. [3] Зельманов Е. И. // Изв. АН СССР. Сер. матем. 1991. Т. 36. № 1. С. 41-66. [4] Зельманов Е. И. // Матем. сб. 1992. Т. 72. № 2. С. 543-565. [5] Струнков С. П. // Изв. АН СССР. Сер. матем. 1990. Т. 54. № 5. С. 1108-1111.

Московский инженерно-физический институт;

Принято редколлегией Chinese University of Hong Kong

E-mail: strunkov@ium.ips.ras.ru kpshum@math.cuhk.edu.hk

24.12.1997 